\title{
PULSATILE FLOW IN CORONARY BIFURCATIONS FOR DIFFERENT STENT- ING TECHNIQUES
}

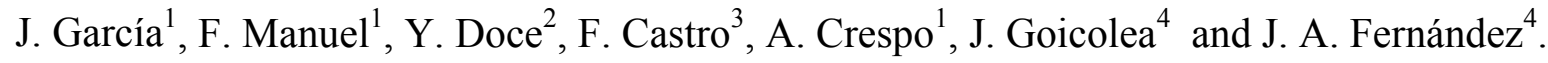

${ }^{1}$ Department of Energy and Fluid Mechanics Engineering. Universidad Politécnica de Madrid, Spain. (garcia@etsii.upm.es)

${ }^{2}$ Department of Mechanics. Universidad Politécnica de Madrid, Spain.

${ }^{3}$ Department of Energy and Fluid Mechanics. Universidad de Valladolid, Spain.

${ }^{4}$ Hospital Universitario Puerta de Hierro. Majadahonda, Spain.

\begin{abstract}
The objective of this work is to analyze the local hemodynamic changes caused in a coronary bifurcation by three different stenting techniques: simple stenting of the main vessel, simple stenting of the main vessel with kissing balloon in the side branch and culotte. To carry out this study an idealized geometry of a coronary bifurcation is used, and two bifurcation angles, $45^{\circ}$ and $90^{\circ}$, are chosen as representative of the wide variety of real configurations. In order to quantify the influence of the stenting technique on the local blood flow, both numerical simulations and experimental measurements are performed. First, steady simulations are carried out with the commercial code ANSYS-Fluent, and then, experimental measurements with PIV (Particle Image Velocimetry) obtained in the laboratory are used to validate the numerical simulation. The steady computational simulations show a good overall agreement with the experimental data. Second, pulsatile flow is considered to take into account the transient effects. The time averaged wall shear stress, oscillatory shear index and pressure drop obtained numerically are used to compare the behavior of the stenting techniques.
\end{abstract}

Keywords: stent, coronary bifurcation, computational fluid dynamics, wall shear stress.

\section{INTRODUCTION}

Flow through severely atherosclerotic vessels is of great interest. Narrowing of a blood vessel, or stenosis, can result from substantial plaque deposit, and may cause a severe reduction of blood flow. These stenosed vessels pose grave health risks and are a major cause of mortality in the industrialized world. The appearance and progression of the atherosclerotic disease depend on individual characteristics, determined genetically, on the presence of risk factors and on local factors related to the vascular anatomy and hemodynamic variables. The consequences of local variations in the flow have been studied in different experimental mod- 
els (Benard et al., 2003). Most of them suggest a correlation between low endothelial shear stress and wall thickness, although there is discrepancy in the results (Zarins et al., 1983; Gnasso et al., 1997 and Shaaban et al., 2000). Wentzel et al. (2000 and 2003) observed an inverse relationship between the shear stress and the neointimal thickness for coronary arteries. The appearance of low and oscillating wall shear stress appears to coincide with early intimal thickening in the carotid artery ( $\mathrm{Ku}$ et al., 1985) and abdominal aorta (Moore et al., 1994). These local effects are indirectly inferred from the preferential distribution of the atherosclerotic plaque on specific locations, such as bifurcations and curved arteries. Bifurcation coronary lesions are frequent, and present a difficult challenge to the interventional cardiologist, with a high complication rate in both long and short term. Major adverse cardiac events are significantly higher in bifurcation versus non-bifurcation lesions and this is mainly attributable to vessel revascularization.

Nowadays, restenosis after percutaneous dilation is the major drawback of coronary angioplasty. It represents a special form of atherosclerosis due to the healing process secondary to extensive vessel trauma induced after intracoronary balloon inflation. The use of coronary stents may decrease the incidence of this phenomenon. Unfortunately, intra-stent restenosis still occurs in 20-30\% of the cases following the stent implantation. Garcia et al. (2006) proposed a simplified model for the neointimal growth or decrease in stent edges and the change in fluid dynamics parameters over time after stent implantation. They also proposed as a criterion for restenosis the sign of the second derivative of the velocity near the wall. There are also many studies that show that links exist between stent design, implantation strategy and also between wall shear stress and neointimal formation, thrombosis, reendothelialization and restenosis (Al Suwaidi et al., 2000; Balossino et al., 2008; Benard et al., 2003; Berry et al., 2000; Charonko et al., 2009 and Deplano et al., 2004).

A critical situation appears when the atherosclerotic lesions are found within coronary bifurcation, in those cases the implantation technique is not unique, and therefore the different flow patterns can produce different behaviors. Although many clinical works have focused on coronary bifurcations, few numerical or experimental studies can be found about stented coronary bifurcations. A double stent implantation in an idealized bifurcation is considered by Deplano et al. (2004). Williams et al. (2010) studied the local hemodynamic changes caused by main branch implantation and with and without subsequent virtual side branch balloon angioplasty in a representative coronary bifurcation. Hu et al. (2010) investigate the magnitude and distribution of wall shear stress after the classical crush stenting for bifurcation lesions. However, none of previous authors made a comparison between different stenting techniques.

There are many techniques for the treatment of bifurcation lesions. In this work three different techniques are considered: simple stenting in main vessel, simple stent in main vessel and kissing balloon through the side branch, and culotte technique, see Fig. 1. In the kissing balloon an opening is made in the side branch with the inflating balloon. In the culotte 
technique a stent is inserted in the side branch. There are other techniques like crush stenting, similar to culotte technique, that are not contemplated here.

For each stenting technique, both $45^{\circ}$ and $90^{\circ}$ bifurcations are evaluated. Initially, the flow is assumed to be stationary and the results are compared to the experimental data obtained in the laboratory with a PIV equipment, to validate the numerical procedure. After that, the same configurations are analyzed numerically but considering pulsatile flow instead. In order to evaluate the performance of the different stented bifurcations, the wall shear stress, the oscillatory shear index and the pressure drop are taken into consideration. Calculations were made also to estimate the total enstrophy and the second derivative of the velocity near the wall to be considered as additional criteria for evaluating the performance of the different techniques; however they have been omitted here because the results obtained indicated a similar behavior to that of the pressure drop criteria.
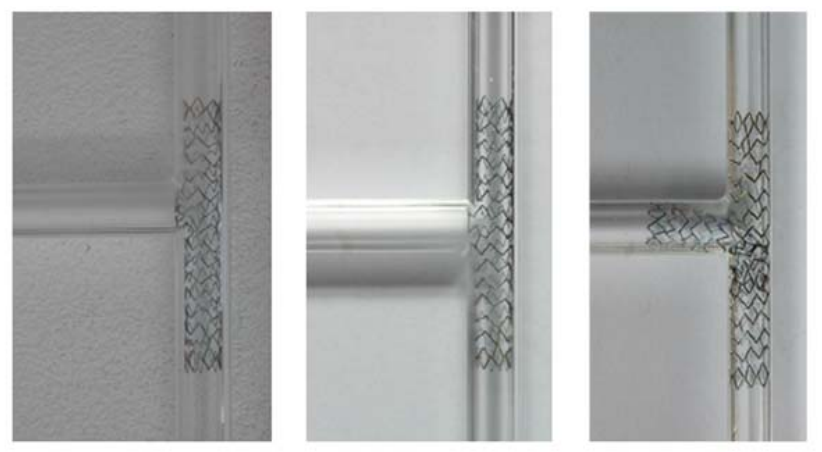

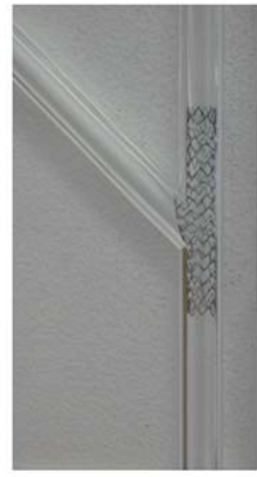

(a)

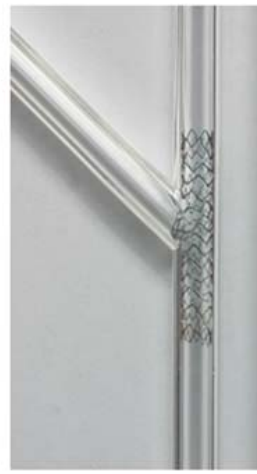

(b)

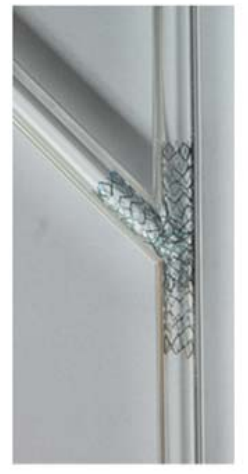

(c)

Figure 1. Stenting techniques considered in this study: (a) simple stenting in the main branch, (b) simple stenting with kissing balloon through the side branch and (c) culotte stenting.

To validate the numerical model it is not necessary to match the experimental model to the physical scaling and characteristics of the coronary vessels. To achieve an accurate simulation of the computational flow, we adopted the same Reynolds number $(\operatorname{Re}=\rho v \mathrm{D} / \mu)$ where $\mathrm{v}$ and $\mathrm{D}$ are characteristic velocity and vessel diameter respectively, $\rho$ the density and $\mu$ the viscosity of the fluid. 


\section{EXPERIMENTAL SET-UP}

Due to the difficulty for experimental studies to match the physical scaling of the coronary vessels, a scaled-up model with full dynamic similarity was created to validate the numerical model.

A hydraulic circuit has been designed to investigate the steady flow in a plexiglass model of a coronary bifurcation. The flow was evaluated using Particle Image Velocimetry. PIV provides instantaneous velocity measurements in a cross section of flow containing reflective microparticles. The technique is based on the comparison of particles position on two images separated by a small amount of time. Reflections and background illumination were blocked using Fluorescent Particles of polymer $(1-20 \mu \mathrm{m})$ in the fluid and a filter to the camera lens. The PIV is a Dantec Flow Map with a double Nd:Yag Laser. The maximum inherent error is of $3 \%$.

To perform the experimental measurements a total of four glass models have been tested for each one of the two bifurcation angles considered, $45^{\circ}$ and $90^{\circ}$. In three of them there are one or two stents, depending on the stenting technique: simple stent, kissing balloon or culotte. The fourth one was tested without stent and was considered the base case, representing a healthy coronary bifurcation. The arteries are represented by straight cylinders of 8 $\mathrm{mm}$ inner diameter. This value of the inner diameter is big for a coronary artery and has been chosen to obtain a better experimental resolution. Therefore, instead of coronary stents, peripheral ones from Biotronik, have been employed. In Figure 1 the experimental models for $45^{\circ}$ and $90^{\circ}$ bifurcations are shown. The working fluid is a mixture of glycerine $(60 \%)$ and water $(40 \%)$ with a viscosity and density similar to that of human blood $(v=3.72 \times 10-6$ $\mathrm{m} 2 / \mathrm{s}, \rho=1099.3 \mathrm{~kg} / \mathrm{m} 3)$.

The flow rate was measured by a gravimetric flow rate. The flow rate was scaled to maintain the same Reynolds number used in the numerical calculations where a typical inner diameter value of $3.2 \mathrm{~mm}$ for a coronary artery is used. Only steady cases are measured in the laboratory. In Figs. 3 and 4, the non-dimensional velocity contours obtained experimentally are compared to the numerical results described in the next section.

\section{NUMERICAL MODEL}

\subsection{Steady cases}

The same geometrical configurations tested in the laboratory, and commented previously, are solved numerically with the commercial code ANSYS-Fluent, but with more realistic dimensions. The inner diameter in the simulations is $3.2 \mathrm{~mm}$ and the length of the stents is 
$23.6 \mathrm{~mm}$. A polyhedral mesh is used, refined near the struts of the stents to capture the corresponding geometry. The mesh has 3 to 5 million of cells depending on the stenting technique. The arterial wall is considered as rigid. This is a reasonable assumption for regions where a percutaneous intervention is made, due to the metallic struts of the stent and the existing plaque in the vessels. The fluid is assumed to be Newtonian with a viscosity $v=3.72 \times 10-6$ $\mathrm{m} 2 / \mathrm{s}$ and density $\rho=1099.3 \mathrm{~kg} / \mathrm{m} 3$, (Reynolds number equal to 172). For the steady simulations, at the inlet a constant velocity equal to $0.2 \mathrm{~m} / \mathrm{s}$ is imposed and at the outlets a zero gauge pressure boundary condition is specified. In order to avoid perturbations in the solution caused by the boundary conditions at the outlets, a bigger computational domain is chosen to perform the steady simulations. In these first configurations, the outlets were located 35 diameters downstream the bifurcation.

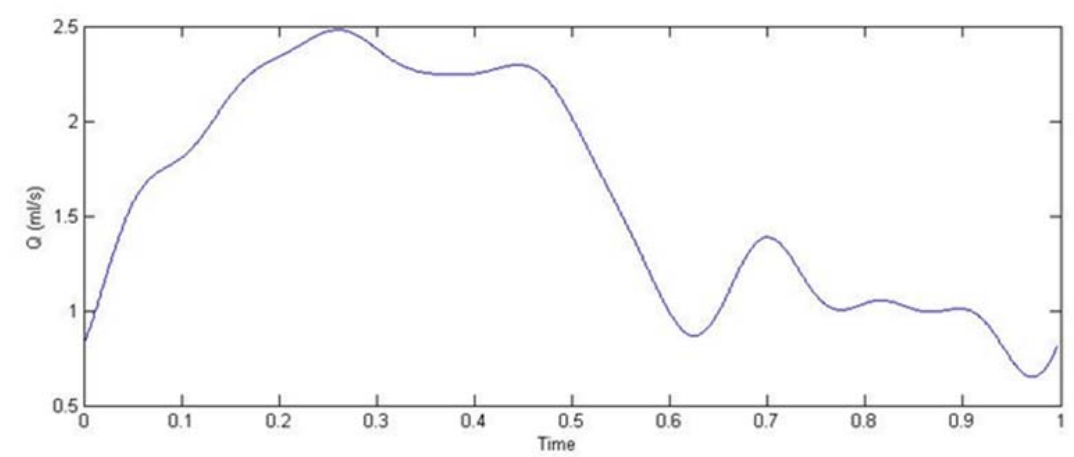

Figure 2. Flow rate waveform versus dimensionless time used in the transient simulations. Taken from Williams et al., 2010.

\subsection{Unsteady cases}

For the unsteady case the velocity is imposed at both entrance and exits. A timevarying parabolic profile is imposed at the inlet. The inlet blood flow velocity waveform, corresponding to resting conditions in a coronary artery, was taken from the literature (Williams et al., 2010), see Fig. 2 for the first period. Only its first 10 Fourier modes were considered in this analysis. The Womersley number corresponding to this flow is equal to 2.2. For the exits, it has been supposed that the ratio of the flow rates at the main and side outlets for the different stenting techniques and bifurcations, are those obtained from steady simulations as shown in Table 1. Four cardiac cycles were simulated to obtain a stable solution; all the results shown in this work correspond to the last cycle. 


\section{RESULTS}

\subsection{Stationary case, numerical and experimental results}

The results shown in this section are referred to the stented region only, or equivalent region for the cases where the stent is not present.

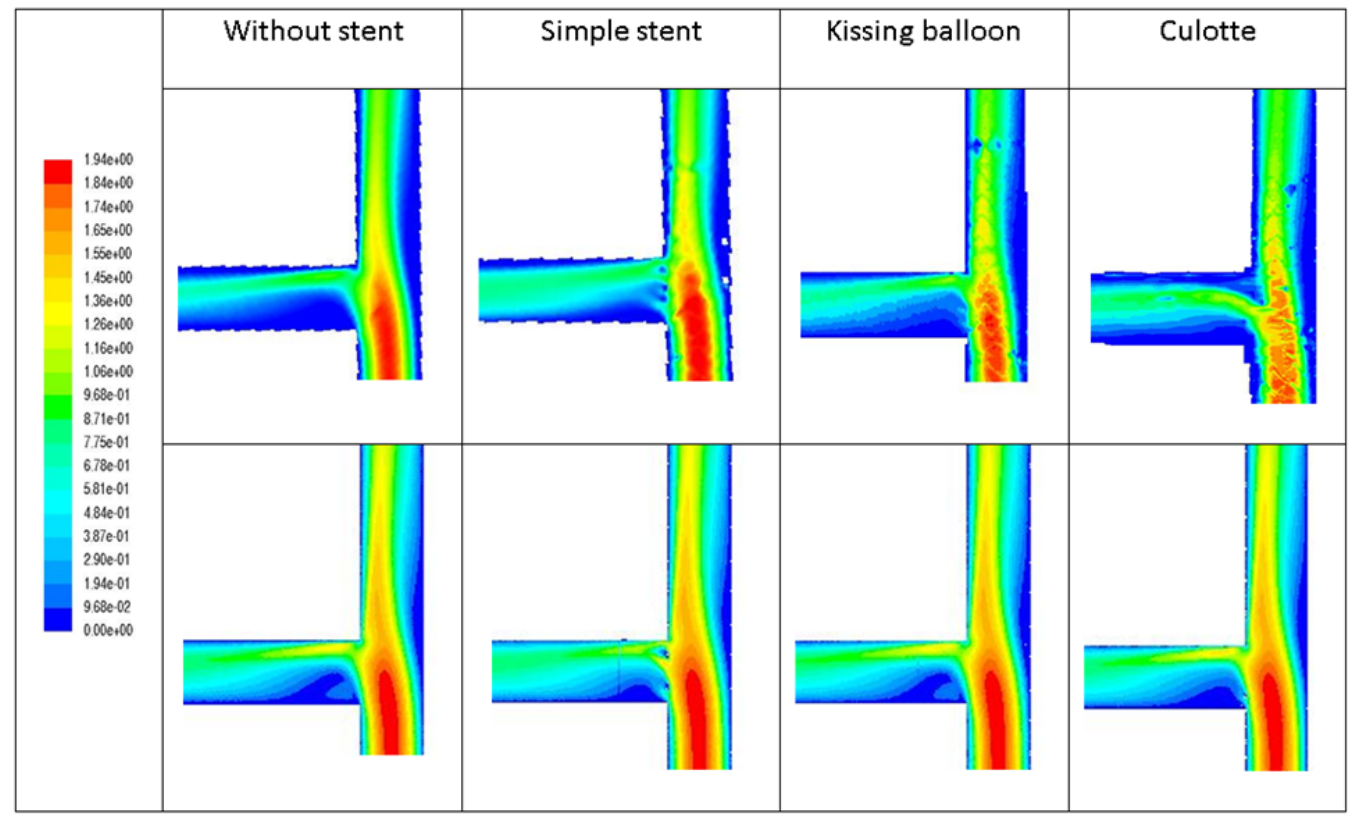

Figure 3. Contours of velocity magnitude obtained with PIV (upper images) and those obtained numerically (lower images) for the base case (without stent), simple stent, kissing balloon and culottte technique for a $90^{\circ}$ bifurcation.

In Figs. 3 and 4 are shown the velocity contours obtained both numerically and experimentally. It can be observed that the flow pattern is more perturbed by the simple stenting technique, where the flow in the side branch is more uniformly distributed downstream due to the presence of the struts. This behaviour is more noticeable for the numerical results of the $45^{\circ}$ bifurcation (Fig. 3), producing a smaller recirculation and stagnant region in the side branch (see also Fig. 4), and consequently smaller areas of low wall shear stress. This tendency is also observed for the transient simulations commented below. Comparing Figs. 3 and 4 it can be seen that the flow pattern depends mainly on the geometrical configuration adopted for the bifurcation and not on the stenting technique used. In the culotte technique, the stent through the side branch may not be completely adjusted to the wall at the flow divider, originating a low velocity region in that area. This effect is not well reproduced numerically due to small differences in the geometrical reconstruction of the distorted struts in that zone. However, the overall agreement between experimental and numerical results is reasonably good. This agreement could be interpreted as preliminary validation of the non-steady results. 


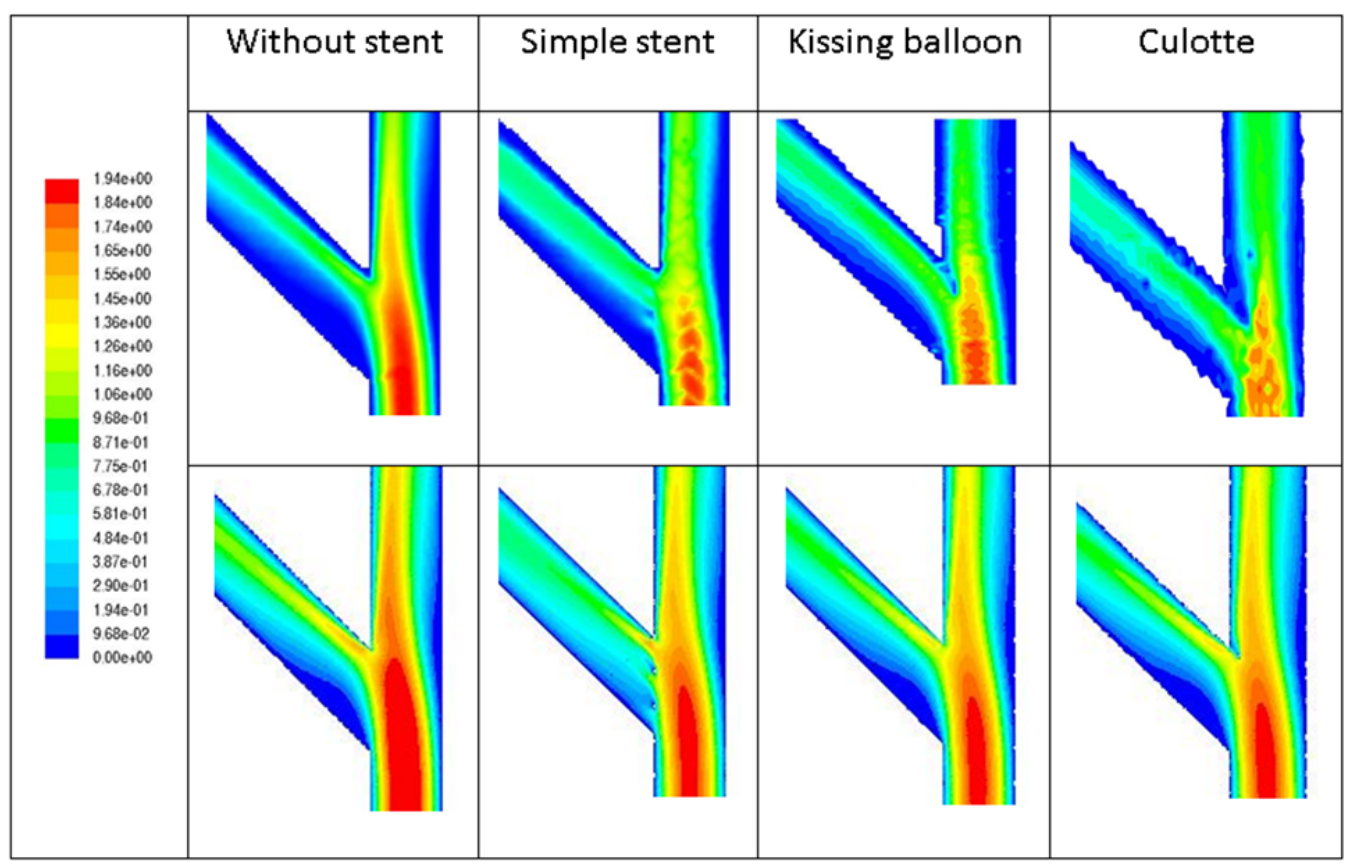

Figure 4. Contours of velocity magnitude obtained with PIV (upper images) and those obtained numerically (lower images) for the base case (without stent), simple stent, kissing balloon and culotte technique for a $45^{\circ}$ bifurcation.

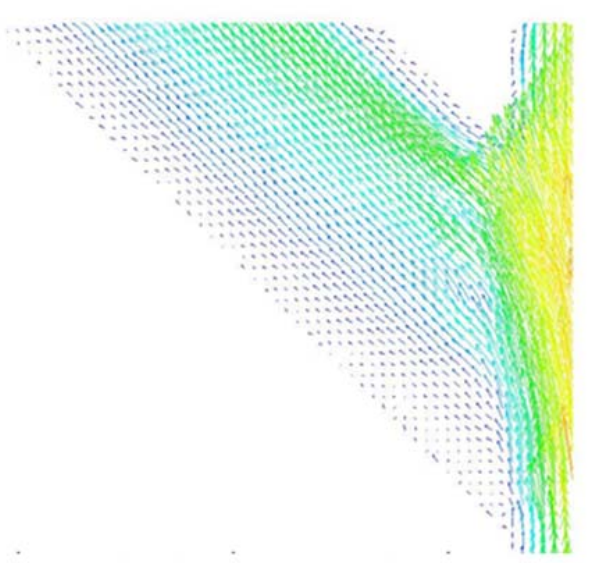

(a)

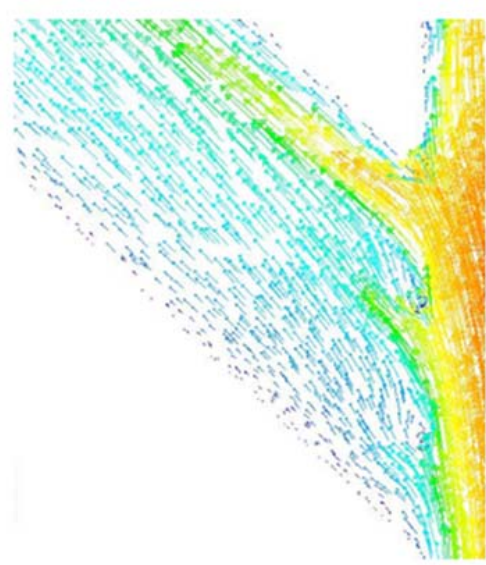

(b)

Figure 5. Velocity vectors in the middle plane for a $45^{\circ}$ bifurcation with a simple stent implantation. (a) Experimental results obtained with PIV and (b) numerical results.

\subsection{Unsteady simulations and comparison of the different stenting techniques}

To evaluate the behaviour of the different stent implantation strategies the following results, obtained from the unsteady calculations, were considered: 
- The pressure drop within the bifurcation.

- The extent of the areas with a time average wall shear stress lower than $0.4 \mathrm{~Pa}$.

- The oscillatory shear index (OSI).
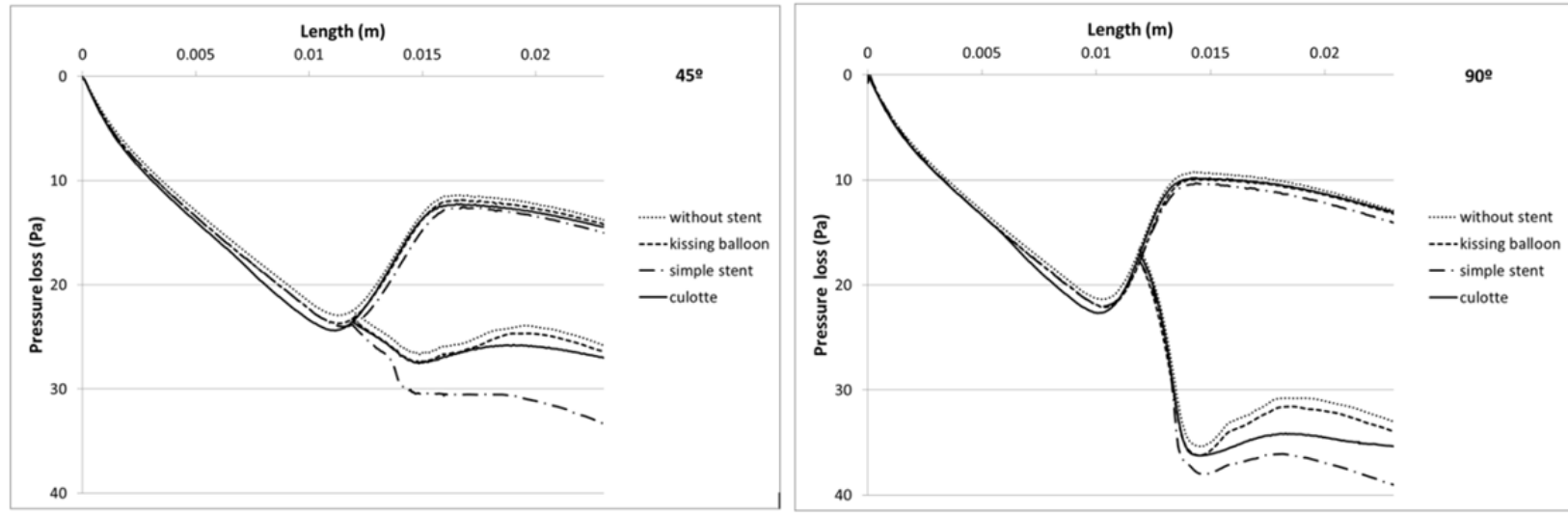

Figure 6. Time average pressure drop within a $45^{\circ}$ (left) and $90^{\circ}$ (right) bifurcation for the four cases studied in the unsteady simulations.

In Fig. 6 the time average pressure loss within the stented region is presented. The pressure losses are larger for $90^{\circ}$ than for $45^{\circ}$, as it can be expected. It can be also seen that in both cases, $45^{\circ}$ and $90^{\circ}$, the pressure drop differences in the main vessel are small for all the configurations, the differences appear in the side branch, where the simple stenting technique produces the worst behaviour, particularly for $45^{\circ}$ where the pressure drop is about $10 \%$ bigger than the one corresponding to the other techniques. The kissing balloon technique is better in all cases. The pressure drop could be considered as a fundamental criterion for drug eluting stents, where the restenosis would be more limited and the reestablishment of the blood flow would be the main objective.

Table 2. Areas $\left(\mathrm{mm}^{2}\right)$ where the time average wall shear stress is lower than $0.4 \mathrm{~Pa}$ for the different stenting techniques.

\begin{tabular}{|c|c|c|c|c|}
\hline & Without stent & Simple stent & Kissing balloon & Culotte \\
\hline $90^{\circ}$ & 16.55 & 13.08 & 17.98 & 39.75 \\
\hline $45^{\circ}$ & 25.73 & 16.65 & 29.09 & 44.68 \\
\hline
\end{tabular}


In Table 2 are presented the areas, in $\mathrm{mm}^{2}$, where the value of the wall shear stress is lower than $0.4 \mathrm{~Pa}$. This value is $20 \%$ of the one corresponding to the Poisseuille solution in the proximal region. The low shear stress regions would be, in principle, the most prone to restenosis. According to these results the culotte technique would be the worst. The simple stent would present globally the better performance. The areas of low wall shear stress are localized at the exterior of the bifurcation, where two recirculation zones appear, see Fig. 7.

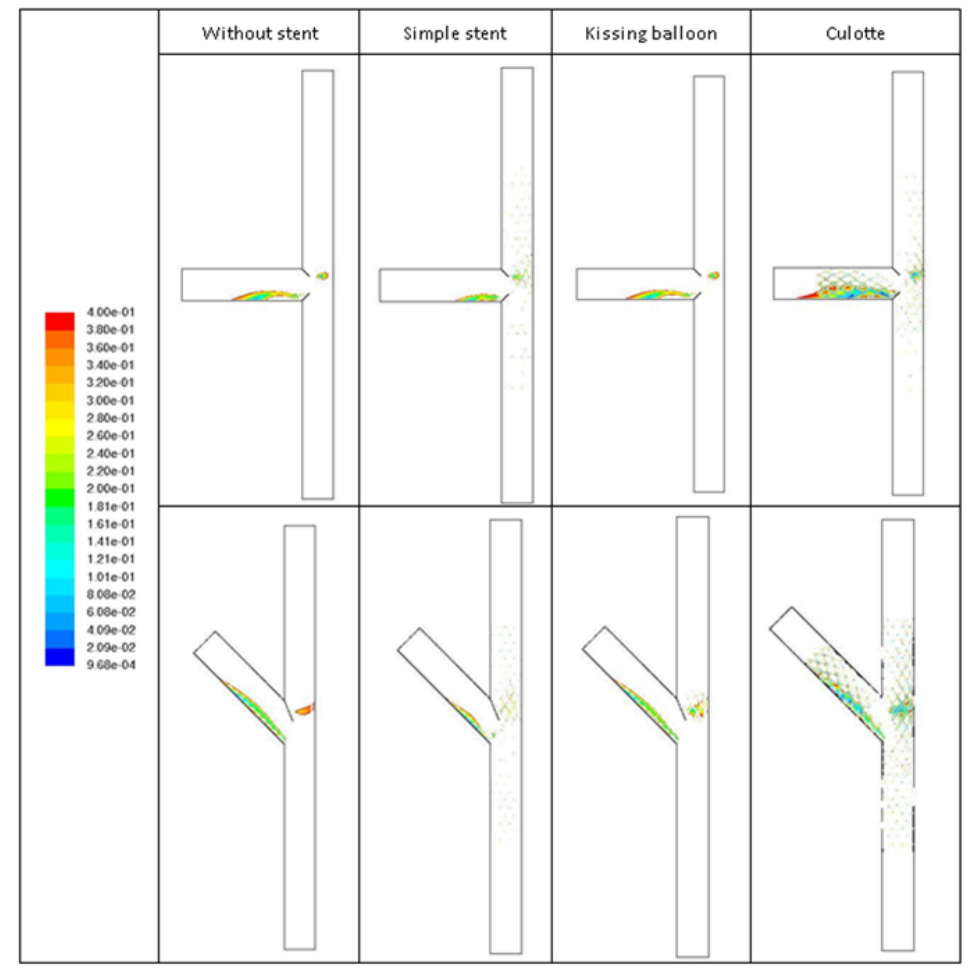

Figure 7. Regions where the time average wall shear stress is lower than $0.4 \mathrm{~Pa}$ for the different stenting techniques.

Obviously, a larger number of stent struts produces larger regions of recirculation behind the struts, this effect could explain why the culotte technique implantation presents the largest value of low wall shear stress area. On the other hand, in the simple stent implantation the struts in the middle of the side branch inlet reduce the size of recirculation bubble.

In Fig. 8, the time evolution of the zones affected by low wall shear stress is shown together with the corresponding velocity magnitude in the middle plane. The time is normalized with the cardiac cycle period. 


\begin{tabular}{|c|c|c|c|c|c|}
\hline & $t /=0.1$ & $t /=0.3$ & $t /=0.5$ & $t /=0.7$ & $t / T=1$ \\
\hline 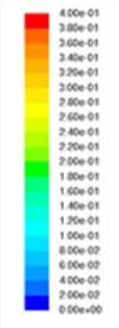 & 1 & & & 4 & \\
\hline \begin{tabular}{ll|} 
\\
\end{tabular} & & & & L & \\
\hline
\end{tabular}

Figure 8. Time evolution of the regions where the wall shear stress is lower than $0.4 \mathrm{~Pa}$ (upper images) and velocity magnitude $(\mathrm{m} / \mathrm{s})$ contours in the middle plane (lower images), for a $45^{\circ}$ bifurcation with culotte technique.

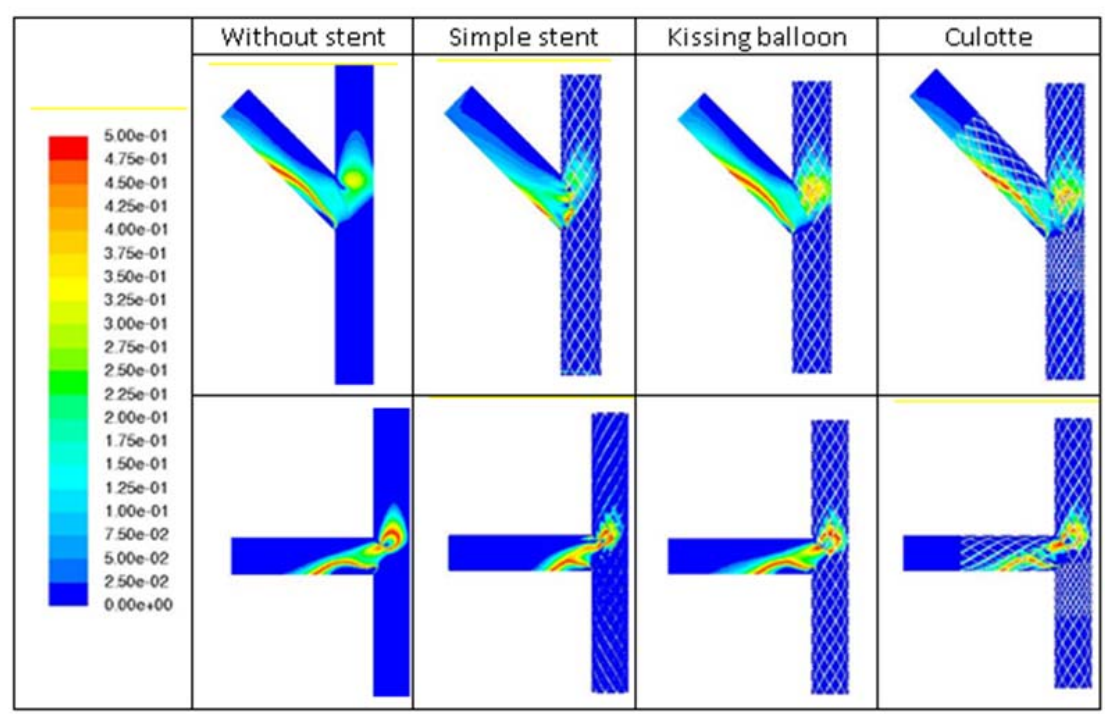

Figure 9. Oscillatory shear index (OSI) for the different configurations.

To take into account the pulsatile nature of the flow the oscillatory shear index (OSI) has been also quantified. It is defined as: 


$$
O S I=\frac{1}{2}\left(1-\frac{\frac{1}{T} \int_{0}^{T} \tau_{w} d t \mid}{\frac{1}{T} \int_{0}^{T}\left|\tau_{w}\right| d t}\right)
$$

where $\mathrm{T}$ is the period of the cardiac pulse and $\tau_{w}$ is the wall shear stress projected in the main flow direction.

It is well known from clinical investigations that restenosis may occur not only in regions of low wall shear stress but in that zones where the flow recirculates, or reversed flow appears. These regions are characterized by temporal oscillations in the direction of the wall shear stress. In Table 3, the area exposed to elevated OSI $(>0.1)$ is shown. When there is a stent located in the side branch the presence of the struts produces a smaller area of recirculating flow and elevated OSI, see Fig. 9. This explains why the area with OSI $>0.1$ is higher for the culotte than the one corresponding to simple stent implantation. This tendency is more marked for the $45^{\circ}$ bifurcation. The best technique turns out to be again the simple stenting because reduces the separation bubble in the side branch.

Table 3. Area $\left(\mathrm{mm}^{2}\right)$ exposed to elevated OSI (OSI >0.1)

\begin{tabular}{|c|c|c|c|c|}
\hline OSI & Without stent & Simple stent & Kissing balloon & Culotte \\
\hline $90^{\circ}$ & 38.51 & 29.69 & 42.01 & 35.04 \\
\hline $45^{\circ}$ & 73.87 & 46.17 & 79.90 & 62.83 \\
\hline
\end{tabular}

\section{CONCLUSIONS}

A numerical and experimental study of the influence of different stenting techniques in a simplified coronary bifurcation is made. The experimental results obtained with PIV for steady cases show a good overall agreement with the numerical ones, validating the computational procedure. In the obtained results, the bifurcation angle has a great influence in the hemodynamic behaviour of different stenting techniques. Simple stenting technique shows the worst results in terms of pressure drop. This criterion could be applied for drug eluting stents where the restenosis is not as critical as the reestablishment of the blood flow. Considering the criteria that evaluate the regions of low wall shear stress $(<0.4 \mathrm{~Pa})$ and elevated OSI $(>$ 0.1 ), in general, the culotte procedure that increases the number of struts shows a worse performance and therefore could increase the restenosis. The best option, in this case, would be the simple stenting. 
Opposite recommendations are obtained depending on the criteria chosen. Due to the struts at the entrance of the side branch, the simple stenting technique creates additional vorticity that leads to higher pressure losses, whereas the regions of low wall shear stress and recirculating flow near the proximal wall of the side branch are reduced.

Obviously the present simulations focus only on the hemodynamic aspects and other factors should be taken into account before choosing the most appropriate stenting technique. Moreover, even if only fluid dynamic criteria are considered, it should be noticed that the configurations studied are idealized coronary bifurcations. The behaviour patterns obtained in this work should be verified in more realistic geometries.

\section{Acknowledgements}

This research was supported by the Spanish Ministry of Science and Innovation under grant DPI2008-03399 and the grant GR57 of JCYL.

\section{REFERENCES}

[1] Al Suwaidi, J., P.B. Berger, C.S. Rihal, K.N. Garratt, M. R. Bell, H.H. Ting, J.F. Bresnahan, D.E. Grill and D.R. Holmes, (2000). Inmediate and long-term outcome of intracoronary stent implantation for true bifurcation lesions. J. Am. Coll. Cardiol. 35:929-936.

[2] Balossino, R., Gervaso, F., Migliavacca, F. and Dubini, G. (2008). Effects of different stent designs on local hemodynamics in stented arteries. Journal of Biomechanics, 41; pp. 1053-1061.

[3] Benard, N., Coisne, D., Donal, E. and Perrault, R. 2003. Experimental study of laminar blood flow through an artery treated by a stent implantation: characterization of intra-stent wall shear stress. Journal of Biomechanics 36, 991-998.

[4] Berry, J.L., Santamarina, A., Moore, J.E. Jr., Roychowdhury, S. and Routh, W.D. (2000). Experimental and Computational Flow Evaluation of Coronary Stents. Annals of Biomedical Engineering, Vol. 28, pp. 386-398.

[5] Charonko, J., Karry, S., Schmieg, J., Prabhu, S. and Vlachos, P. (2009). In vitro, TimeResolved PIV Comparison of the Effect of Stent Design on Wall Shear Stress. Annals of Biomedical Engineering, vol. 37, No. 7, pp. 1310-1321.

[6] Deplano, V., Bertolotti, C. and Barragan, C. (2004). Three-dimensional numerical simulations of physiological flows in a stented coronary bifurcation. Medical \& Biological Engineering \& Computing, Vol. 42, pp. 650-659.

[7] Garcia, J., Crespo, A., Goicolea, J., Sanmartín, M. and García, C. (2006). Study of the evolution of the shear stress on the restenosis after coronary angioplasty. Journal of Biomechanics, 39, pp. 799-805.

[8] Gnasso, A., Irace, C., Carallo, C., De Franceschi, M.S., Motti, C., Mattioli, P.L., Pujia, A., 1997. In vivo association between low wall shear stress and plaque in subjets with asymmetrical carotid atherosclerosis. Stroke 28 (5), 993-998.

[9] Ku, D.N., Giddens, D.P., Zarins, C.K., Glagov, S., (1985). Pulsatile flow and atherosclerosis in the human carotid bifurcation: positive correlation between plaque location and 
low and oscillating shear stress. Atherosclerosis 5 (3), 293-302.

[10] Moore, J.E., Xu, C., Glagov, S., Zarins, C.K., Ku, D.N., 1994. Fluid wall shear stress measurements in a model of the human abdominal aorta: oscillatory behavior and the relationship to atherosclerosis. Atherosclerosis 110, 225-240.

[11] Shaaban, A. M., Duerinckx, A.J., 2000. Wall shear stress and early atherosclerosis: a review. AJR American Journal of Roentgenol 174 (6), 1657-1665.

[12] Wentzel, J. J., Whelan, M.D., van der Giessen, W.J., van Beusekom, H.M., Andhyswara, I., Serruys, P.W., Slager, C.J., Krams, R., 2000. Coronary stent implantation changes 3-D vessel geometry and 3-D shear stress distribution. Journal of Biomechanics $33,1287-1295$.

[13] Wentzel, J. J., Gijsen, F.J.H., Stergiopulos, N., Serruys, P.W., Slager, C.J. Krams, R., 2003. Shear stress, vascular remodeling and neointimal formation. Journal of Biomechanics 36, 681-688.

[14] Williams, A.R., Koo, B.K., Gundert, T.J., Fitzgerald, P.J. and LaDisa, J.F. 2010. Local hemodynamic changes caused by main branch stent implantation and subsequent virtual side branch balloon angioplasty in a representative coronary bifurcation. J. Appl. Physiol., 109:532-540.

[15] Zarins, C.K., Giddens, D.P., Bharadvaj, B.K., Sottiurai, V.S., Mabon, R.F., Glagov,S., 1983. Carotid bifurcation atherosclerosis. Quantitative correlation of plaque localization with flow velocity profiles and wall shear stress. Circulation Research, 53 (4), 502-514. 